\title{
Role of Status in Group Processes
}

Edward J. Lawler

Cornell University

Research on Managing Groups and Teams (2005), 7, 315-325 


\begin{abstract}
This chapter organizes the other chapters of the volume around a fundamental statusaffirmation principle, namely, that status differentials generate corresponding differences in performance expectations which, in turn, produce behaviors that affirm performance expectations. The chapters in this volume elaborate that proposition by showing how information exchange, patterns of privilege, and the accuracy of power perceptions reflect or strengthen the status-affirmation process. Several chapters also suggest conditions that forestall or weaken this process such as claims to expertise and communication styles. Other chapters can be construed as offering applications of the status-affirmation principle to the performance of corporate project teams and to the relationships between standard and nonstandard employees in the workplace. Overall, the chapters reflect the strength and vitality of the tradition of work on group processes.
\end{abstract}


The importance of groups to human experience and interaction is undeniable and incontrovertible. People live their lives in groups, in the workplace, in families, among friends, in neighborhoods, and in communities. Groups or group affiliations are important sources of identity, cooperation, collaboration, and a sense of belonging. Such effects exemplify positive consequences of group affiliations for individuals and organizations. Groups or group affiliations also are a source of negative consequences such as exclusion, injustice, discrimination, and violence. Still other group phenomena may have either positive or negative consequences in these terms. A conflict may promote collaborative relations and unity or discrimination and hostility, and the same can be said for a host of other phenomena. The upshot is that groups are a source of both order and disorder. On the one hand, group affiliations promote and organize social interactions, bringing together individuals with similar goals; on the other hand, groups generate divisions or differentiation that foster dissatisfaction, dissent, and conflict.

Status and status processes are fundamental to these "order producing” and "disorder producing” effects of group affiliations and relations. Status structures facilitate order by “expressing” or manifesting prevailing definitions of worth, value, and competence. Those of higher status are perceived (accurate or not) as higher on these dimensions. Status differentiation facilitates disorder by raising issues of justice/injustice, equal opportunity, and legitimacy. The chapters of this conference move the field of group processes forward by addressing these varied effects of status relations and processes.

The work here represents an important tradition of group process research that originated primarily in business schools over the last 20 or so years. I would term this the "management tradition.” It draws heavily on theories from psychology (e.g., social identity theory) and, more recently, sociology (e.g., expectation states theory), but it is distinctive in at least three 
interrelated ways: The substantive focus is on problems and issues relevant to the workplace or work organizations; it is directed at addressing problems more than at building theory; and, it is more explicitly interdisciplinary than the group processes traditions found in psychology and sociology. These features of the "management tradition" are an important source of its research contributions to the "group processes" area as a whole. The workplace focus gives the tradition a theme, the problem focus around this theme gives it more explicit applied or practical value, and the interdisciplinary nature of the tradition gives it a refreshing intellectual openness and breadth. The chapters of this volume reflect these strengths quite well and suggest the vitality of this tradition.

\section{BACKGROUND}

Fundamental changes in the world suggest that a deep and subtle understanding of status processes in groups is increasingly important to most areas of human experience. Part of the reason is a general decline in hierarchy as a mechanism of control and for accomplishing collective or organizational goals. This is manifest in more collaborative work systems, an increase in the importance of negotiation and trust across groups within organizations, and so forth. In the workplace of today, collaborative relations linking actors in different geographic locations, from different social backgrounds, and with different experiences are critical parts of job responsibilities and essential for accomplishing organizational goals or objectives. These differences, however, have status implications. Similarly, changes in the family put more premium on shared responsibilities and the coordination and negotiation of tasks, most of which respond to and attempt to overcome gender status differentiation. Families have become more 
collaborative and less hierarchical, as have workplaces. Finally, the decline of formal memberships in organizations (e.g., the "bowling alone” phenomenon), the growing importance of networks of weak ties for professional success, and the changing nature of careers all point to the importance of groups as key social mechanisms. These give actors a sense of belonging that promotes career opportunities, constrains unethical behavior, and fosters commitments to larger social units shared by substantial populations of people. The most important aspects of group phenomena today are not necessarily the formal, highly structured features, but rather informal, ties, networks, and associations that "come and go” and have revolving memberships and everchanging objectives. In this context, the informal processes by which status differentiation is created, enacted, and reproduced are even more central as research problems. This volume identifies and fleshes out several of these important informal aspects and dimensions of status in work settings.

Most all of the chapters in this volume conceive of status as involving social evaluations of worth or competence across individuals in a group or across groups within an organization. Such evaluations are the basis for ranking individuals, structuring responsibilities, and defining formal or informal positions. The evaluations can involve social categories (e.g., gender, age, race, ethnicity) that have cultural beliefs and expectations associated with them, group affiliations or memberships that bear on the perceptions of individual members (e.g., group identities), or particular performances that suggest relevant competence or expertise (e.g., education, prestige of school). Expectation States Theory in sociology (Berger, Fisek, Norman, \& Zelditch Jr. 1977; Ridgeway \& Walker, 1995) has distinguished “diffuse” status characteristics, which are based on cultural beliefs about worth (Ridgeway, Boyer, Kuipers, \& Robinson, 1998), from specific status characteristics, which stem from demonstrated competence 
on some task. Status characteristics, whether diffuse or specific, promote patterns of deference, compliance, and conformity. The chapters of this volume suggest important elaborations of how and when status differentiation has positive or negative effects on group relations and success.

\section{STATUS-AFFIRMATION PROCESS}

A fundamental principle, that I will term "status affirmation,” can serve as a backdrop for analyzing the implications of the work herein. The "principle of status affirmation" specifies and interconnects three "moments" in a status process:

(1) Status differences (i.e., differences in social evaluation) create differential performance expectations. Interpreted broadly, “performance expectations” include not only individual task performance, but also group performance (i.e., likelihood of being a good team member, contributing to the collective good, and giving priority to group over individual interests if necessary).

(2) Performance expectations generate affirming behaviors on the part of a focal individual and others with whom they are interacting. People act on differential performance expectations by behaving differentially toward those with different status (evaluations).

(3) The affirming behaviors, in turn, strengthen and/or reproduce the social evaluations underlying the status differences. I would argue that this "status-affirmation process" is one of the most well-founded principles found in the social sciences. It has been theorized carefully and researched substantially and could be construed as 
approximating a "basic law” of social interaction. The principle articulates in a simple way how and why status differences generate regular, predictable, and orderly patterns of interaction, how and why such differences are resilient, self-fulfilling prophecies, and why they may generate, under some conditions, a sense of injustice and behavioral efforts to change an existing status relationship.

Using the principle of status affirmation as a backdrop, the chapters of this volume can be grouped under three categories:

(1) those that elaborate the status-affirmation principle,

(2) those that offer strategies for mitigating status affirmation, and

(3) those that apply this principle to another group phenomenon.

Each of these categories is discussed, in turn, below.

\section{ELABORATIONS}

The Chen and Lo chapter emphasize the differential expectations embedded in individualist and collectivist cultures. Individualist cultures draw attention to intragroup differences and associated expectations, whereas collectivist cultures draw attention to intergroup expectations. Thus, “performances” around which expectations form are culturally grounded constellations that are embedded in the social context. This implies a broader conception or interpretation of “performance” and "performance expectations” than often found 
in the research literature on groups, especially by American Social Psychologists and Sociologists. What status-relevant information becomes salient (e.g., intragroup versus intergroup differences) in a given interaction or group setting is contingent on themes of the larger culture.

Rosette and Thompson provide a review and theoretical analysis of how privileges based on ascribed (unearned) statuses can become perceived as achieved (earned). This is termed a “camouflage effect.” Privilege is defined as additional or ancillary “rewards and advantages” that are accorded to those in social categories or positions that yield status. The argument is that when high-status persons receive a privilege, it is viewed as earned, whereas when low-status persons are given a privilege it is viewed as unearned. An example is how the public responds to special consideration given to legacies versus under-represented minorities in college admissions. This chapter suggests that in work organizations, privilege is awarded on the basis of ascribed characteristics, but socially defined as achieved and deserved by those who receive such privileges. They interpret these effects as due to the threat to self-identity or self-esteem and the expectations associated with high-status positions (Berger et al., 1977). This chapter suggests that higher status, not only has "affirming effects" through expectations and related behavior, but also "status enhancing” effects that stem from adding privileges that enhance performance expectations.

Wittenbaum and Bowman offer several propositions about how status affects information exchange in groups. Status affects the patterns of information sharing, the nature of the information shared, and also the evaluation of the person doing the information sharing. They suggest that high-status members generally will communicate more information, shared and unshared; information that is unique will be valued more and remembered more if communicated 
by high-status members; and there is a general bias in favor of shared information because it is status enhancing, and this pattern is stronger for low-status persons. Patterns of information exchange tend to reflect and affirm status structures.

The chapter by Overbeck, Correll, and Park posits a sorting mechanism through which new, undifferentiated groups develop status hierarchies. Among heterogenous status seekers, hierarchy emerges rather easily, but among homogenous status seekers, a sorting mechanism moves some upward and others downward. Such conditions put a premium on interpersonal skills and sensitivity. Those that achieve more status in homogenous groups are likely to have a collective orientation and more concern for others, whereas in heterogeneous groups, an individualistic orientation is dominant. One implication is that there are evolutionary processes that generate hierarchy without the mediation of expectations. This chapter may help explain how positions with differentiated statuses develop initially and, therefore, unleash the process specified by the status-affirmation principle.

Two chapters build power into the analysis, though without explicit ties to status. Cameron, Spataro, and Galinsky develop the notion that the perception of power is as important as the actual power people have. A highly powerful person may not be perceived as having power and a person with low power may be perceived as having much more power than they in fact have. People develop a "sense of power" which is defined as the perception of one’s capability of influencing another. Cameron et al. argue that having an accurate sense of one’s power is important to effective use of power. Mortorana and Galinsky address the question of when lower power persons essentially respond in a subordinate way and reproduce conditions that disadvantage them. This entails a self-fulfilling prophecy that dovetails the statusaffirmation principle. However, Mortorana and Galinsky put forth an emotional explanation for 
this behavior, that is, fear and sadness associated with low power positions generates inaction. Low power individuals resist and seek change when the power hierarchies are unstable, illegitimate, and allow mobility by individuals across levels. A sense of power helps transform fear into anger and related action to change the hierarchy. This also is a mechanism for changing the status structure.

To summarize, elaborations of the status-affirmation principle indicate that patterns of information sharing by low- and high-status persons tend to affirm the status order; individualist versus collectivist cultures make salient intragroup versus intergroup status differences, respectively; status differences are enhanced by patterns of privilege that add to the benefits and perceived worth of those in higher-status positions; and an accurate sense of power may enhance the degree that high-status persons strategically generate the status-affirmation process.

\section{Reducing Status Affirmation}

Three chapters suggest qualifications or ways to weaken or eliminate the statusaffirmation process. Fraidin and Hollingshead suggest how gender stereotypes become "selfreinforcing” by virtue of what tasks are assigned to men and women. Task assignments tend to be shaped by gender stereotypes, that is, gender is perceived as entailing different competencies and thus differential status in particular task situations. Their research empirically demonstrates that "claims of expertise" are a mechanism for women to reduce the effects of gender stereotypes somewhat, and if these are validated by other information on the particular female's background, the claims have an even stronger effect. The important implication is that claims to expertise are 
a strategy for mitigating or eliminating the status-affirming process. Such claims involve a challenge to the existing status order.

The chapter by Fragale shows further that communication style, involving both verbal and nonverbal cues, is an important source of status if adjusted to the context. These effects occur by either conveying “interpersonal abilities” or “intellectual abilities.” Fragale’s research shows that in a finance department within a corporation, more assertive communication styles generate greater status and that this occurs because more assertive speakers are viewed as more competent. Interpersonal abilities play no role in status acquisition here. In a human resources department, however, assertive speech diminishes status because it is interpreted as implying poorer interpersonal abilities. A less assertive style conveys to others greater interpersonal abilities. In an organization with individualist values, persons will confer more status to persons with the more assertive communication styles, whereas within an organization with collaborative values, more status will be conferred upon persons with nonassertive communication styles. The implications are that, to be effective, the assertiveness with which “claims to expertise” are expressed need to fit the values and needs of the organizational context. Together, the Fragale chapter, along with the one by Fraidin and Hollingshead, suggest that those with lower status can acquire more status and influence in work groups and organizations, than their initial status position or ranking suggests, by adopting “appropriate” behavioral styles for the context and by making claims to expertise that will be perceived as valid and justifiable.

De Cremer and Tyler argue that "respect" from others in the group enhances the inclination of a member to contribute to the collective good. For instance, they indicate that when $\mathrm{O}$ conveys respect to $\mathrm{S}, \mathrm{S}$ feels a greater sense of belonging and therefore is more willing to contribute to the group. Respecting behavior communicates a social evaluation that carries 
with it expectations for greater group contributions which S dutifully and willingly enacts. Respect is something that can be given by anybody to anybody, regardless of status position or evaluations. An interesting implication of this study is that mechanisms for generating behaviors that respect others in a group should mitigate the negative consequences of status differentiation with that group. Patterns of respect may not eliminate status differentiation around group tasks, but they presumably would encourage less deference to those with greater status, thereby weakening the status-affirmation process.

The status-affirmation process suggests a strong, inherent tendency for a status structure to be reproduced. The chapters by Fragale, Fraiden, and Hollingshead, and De Cremer and Tyler have implications for how the process can be counteracted by behavioral strategies and patterns. Claims to expertise and mutual patterns of respect are examples. The Wittenbaum and Bowman chapter suggests further that status effects are attenuated if the communication medium reduces the visibility of status cues and enhances the anonymity of individuals’ identities.

\section{Applications}

Two chapters in the conference offer interesting applications of the status- affirmation process. Haas examines the performance of corporate project teams with an international composition. She argues that the distinction between "cosmopolitans" and "locals" on these teams generates status differences such that cosmopolitans participate more, are consulted more, and have greater influence than locals. Where such teams use more external knowledge, status evaluations are consensual, that is, cosmopolitans are perceived by group members as warranting more influence. When such teams use less external knowledge, status rivalry occurs between 
cosmopolitans and locals on the teams. Thus, the task determines whether a status characteristic, that is activated in the situation, has a positive or negative effect on group relations.

Vough, Broshak, and Northcraft interpret the relations between standard (regular) and nonstandard (contract) employees partially in status terms. The effects of nonstandard workers on group relations and performance depend on the competence (performance expectations) of the nonstandard workers. If highly competent nonstandard workers threaten the employment of standard workers status rivalry tends to occur, if standard workers perceive nonstandard workers as a burden, requiring more of their time, they represent a potential threat to the prestige of the work they do in the organization. These effects are stronger if standard and nonstandard employees work side-by-side and if they perform similar tasks. The Haas and Vough et al. chapters reveal distinct categories of employees that can be the basis for status rivalries. They identify conditions under which such rivalries are stronger or weaker, and some of these processes can be interpreted as status-affirmation effects.

\section{CONCLUDING REMARKS}

An implicit message of this volume is that status involves comparisons of general worth and these operate at multiple levels: (a) between individuals (person-to-person), (b) between persons and groups (person-to-group), and (c) between groups (group-to-group). Virtually all of the chapters in this volume incorporate multiple levels in some way. Some focus on one of these levels, but analyze it in the context of another, for example, examining the relation of person-toperson (intragroup) ties to person-to-group ties; others directly contrast conditions that make one level more salient than the other (e.g., intragroup versus intergroup statuses); and still others 
show how dynamics at one level affect processes at another. The notion that status should be interpreted as a multilevel phenomenon is not new, but the chapters of this volume add force and substance to this argument.

To illustrate, De Cremer and Tyler’s chapter analyzes respect as an “intragroup” status, but shows its effect on person-to-group relations. Chen and Lo's chapter indicates that different types of culture shape whether intergroup and intragroup statuses are more salient, and Haas’s use of the cosmopolitan-local distinction can be interpreted similarly. Fraidin and Hollingshead's and Rosette and Thompson's chapters have an intergroup focus involving different "groups" (gender, hierarchical status). A multilevel approach introduces more complexity in analyses of status and puts more demands on our theories. This volume suggests the need for multilevel theories of status that more explicitly and self-consciously bridge and interconnect the levels (see Markovsky, 1997, for discussion of multilevel theorizing). Only one chapter in this volume, Randel, Hoon, and Earley, moves in this direction. Randel et al. take notions of expectation states theory, which generally are focused on dyadic relations, and integrate these with the role identity theory of Sheldon Stryker (1980). Role identity theory can put the status characteristics in a structural context and offers a way of assessing how multiple identities with distinct status implications can vary in importance and salience to actors. Given people interact in the context of multiple identities or roles (i.e., group affiliations), the main argument is that identity salience and commitment determines the status characteristics most likely to become relevant in a given situation. This is a promising theoretical direction for future theorizing on status in the workplace.

As a sociologist, somewhat on the fringe of the "management tradition" of group processes, I see this volume as a reminder of the intellectual fragmentation within the group 
processes area. The lines of contact and communication among scholars in sociology, psychology, and management/OB fields are ad hoc and relatively unsystematic. Distinct collegial institutions within these fields have developed ways to promote intellectual exchange and collaboration around their own core set of problems. In psychology, this generally would be the yearly meetings of the Society of Experimental Social Psychology; in sociology, it would be the yearly Group Processes Conference organized at the meetings of the American Sociological Association, and the annual series, Advances in Group Processes. In the management/OB area, it is these yearly conferences and this annual series, Research on Managing Groups and Teams. It is noteworthy that within the same 15-20-year period, sociologists with an interest in group processes and those in business schools adopted similar strategies for building their areas (i.e., an annual volume and an annual conference). Each tradition has been strengthened and firmly established in their fields through these formal and informal associations. However, these strategies also tend to reduce incentives for or push toward stronger ties and collaborations across the sociologists and management/OB intellectual communities, in particular. Conferences that promote more cross-fertilization across these traditions would likely enrich each and bring more visibility and attention to the general area of group processes. The theoretical focus of the sociologic tradition and the problem focus of the management tradition are complementary and easy to bridge. Scholars in each of the traditions have much to learn from each other. 


\section{References}

Berger, J. M., Fisek, H., Norman, R. Z., \& Zelditch, M., Jr. (1977). Status characteristics and social interaction. New York: Elsevier.

Markovsky, B. (1997). Building and testing multilevel theories. In: J. Szmatka, J. Skvoretz \& J. Berger (Eds), Status, network, and structure (pp. 13-28). Stanford, CA: Stanford University Press.

Ridgeway, C. L., \& Walker, H. A. (1995). Status structures. In: K. S. Cook, G. A. Fine \& J. S. House (Eds), Sociological perspectives on social psychology (pp. 281-310). Needham Heights, MA: Allyn and Bacon.

Ridgeway, C. L., Boyer, E. H., Kuipers, K., \& Robinson, D. T. (1998). How do status beliefs develop? The role of resources and interactional experience. American Sociological Review, 63, 331-350.

Stryker, S. (1980). Symbolic interaction: A social structural version. Menlo Park, CA: Benjamin Cummins. 